\title{
Simultaneous Evaluation of Laryngopharyngeal Reflux and Swallowing Function Using Hypopharyngeal Multichannel Intraluminal Impedance Measurements in Neurologically Impaired Patients
}

\author{
Daisuke Masui, ${ }^{1 *}$ Suguru Fukahori, ${ }^{1}$ Naoki Hashizume, ${ }^{1}$ Shinji Ishii, ${ }^{1}$ Naruki Higashidate, ${ }^{1}$ Saki Sakamoto, ${ }^{1}$ Shiori Tsuruhisa, \\ Hirotomo Nakahara, ${ }^{1}$ Nobuyuki Saikusa, ${ }^{1}$ Yoshiaki Tanaka, ${ }^{1,2}$ and Minoru Yagi ${ }^{1}$ \\ ${ }^{I}$ Department of Pediatric Surgery and ${ }^{2}$ Division of Medical Safety Management, Kurume University School of Medicine, Fukuoka, Japan
}

\section{Background/Aims}

This study aims to evaluate the presence of laryngopharyngeal reflux (LPR) and to investigate the use of hypopharyngeal baseline impedance (BI) for assessing swallowing dysfunction and gastroesophageal reflux disease (GERD) using hypopharyngeal multichannel intraluminal impedance and pH (HMII-pH) monitoring in neurologically impaired patients (NIPs).

\section{Methods}

The study population in this retrospective study comprised $20 \mathrm{NIPs}$ (mean age, $36.1 \pm 15.0$ years; age range, 13-64 years) who underwent multichannel intraluminal impedance and $\mathrm{pH}(\mathrm{MII}-\mathrm{pH}), \mathrm{HMII}-\mathrm{pH}$, and laryngoscopy using the Hyodo scoring method from December 2016 to April 2019. The MII-pH and HMM-pH parameters were compared in the NIPs, whereas hypopharyngeal BI values were compared between NIPs with $\geq 5$ and $<5$ in Hyodo scores. Correlations between the hypopharyngeal BI values and the Hyodo score were analyzed using Spearman's correlation coefficient. A receiver operator characteristic curve was created to determine the optimum cut-off of hypopharyngeal BI value to discriminate SD.

\section{Results}

Three NIPs were diagnosed with pathological LPR and GERD by the HMII-pH monitoring. No significant differences in parameters were observed between MII-pH and HMII-pH monitoring. The correlation analysis demonstrated a significant negative correlation between the hypopharyngeal BI values and Hyodo scores. The optimal cutoff value for hypopharyngeal BI was $1552 \Omega$.

\section{Conclusions}

This study demonstrated the usefulness of HMII-pH monitoring in identifying NIP with pathological LPR. Considering the difficulties in performing examinations in NIPs, HMII-pH monitoring may be a potentially useful technique for the simultaneous evaluation of swallowing dysfunction, LPR, and GERD in NIP.

(J Neurogastroenterol Motil 2021;27:198-204)

\section{Key Words}

Baseline impedance; Dysphagia; Gastroesophageal reflux disease; Laryngopharyngeal reflux; Multichannel intraluminal impedance measurement

Received: June 3, 2020 Revised: None Accepted: January 2, 2021

(.) This is an Open Access article distributed under the terms of the Creative Commons Attribution Non-Commercial License (http://creativecommons. org/licenses/by-nc/4.0) which permits unrestricted non-commercial use, distribution, and reproduction in any medium, provided the original work is properly cited.

*Correspondence: Daisuke Masui, MD, PhD

Department of Pediatric Surgery, Kurume University School of Medicine, 67 Asahi-machi, Kurume, Fukuoka 830-0011, Japan Tel: +81-942-31-7631, Fax: +81-942-31-7705, E-mail: masui_daisuke@med.kurume-u.ac.jp 


\section{Introduction}

Swallowing dysfunction (SWD) and gastroesophageal reflux disease (GERD) have been recognized as critical complications, which occur with high frequency in neurologically impaired patients (NIPs) and cause severe growth retardation and malnutrition, ${ }^{1,2}$ and as the major factors for the occurrence of aspiration pneumonia, the most common cause of death in NIP. ${ }^{3}$ Therefore, appropriately assessing the presence of both SWD and GERD in NIP is vital.

Endoscopic and videofluoroscopic examinations are commonly conducted to assess the presence of SWD and to quantify the risk of aspiration; in addition, the Flexible Endoscopic Evaluation of Swallowing along with the Hyodo scoring method (HSM) are utilized for their ease of use in neurologically normal patients. However, the swallowing function cannot be appropriately evaluated in most NIPs due to their inability to complain about their symptoms and follow instructions. Consequently, some NIPs experience severe aspiration pneumonia, whereas some undergo invasive surgical procedures such as laryngotracheal separation without any appropriate evaluation. Therefore, a more simplified and accurate technique is highly anticipated to assess SWD in NIPs.

Multichannel intraluminal impedance and pH (MII-pH) monitoring has recently emerged as a novel technique to evaluate GERD, irrespective of the $\mathrm{pH}$ value. Until now, we have attempted to investigate and report the pathophysiology of GERD in $\mathrm{NIPs}^{4-7}$ In MII-pH, the baseline impedance (BI) value is dropped when a liquid comes in contact with the 2 adjacent impedance sensors. In particular, a low BI value in the lower esophagus indicates esophageal motility disorders, such as esophageal achalasia; recently, we reported its usefulness in assessing esophageal motility in children with esophageal disorders. ${ }^{8}$ SWD includes physiological limitations during the oral and pharyngeal phases of swallowing. In the pharyngeal phase, inadequate pharyngeal peristalsis generates excessive pooling of food or fluid in the vallecula or pyriform sinuses. ${ }^{9}$ HSM evaluates saliva retention and pharyngeal clearance as important factors of scoring. ${ }^{10,11}$ Therefore, we believe that a low hypopharyngeal BI value indicates the presence of pharyngeal saliva retention, which can be used as an objective indicator of SWD in NIPs.

Laryngopharyngeal reflux (LPR), defined as retrograde flow of gastric contents beyond the esophagus to the larynx and pharynx, has been increasingly recognized over the past 2 decades as a phenomenon distinct from GERD. ${ }^{12,13}$ Moreover, a relationship between LPR and pulmonary diseases, such as idiopathic pulmonary fibrosis and "adult-onset" asthma, has been suggested. ${ }^{14,15}$ Nevertheless, the pathophysiology of LPR is poorly understood. The endoscopic findings of LPR on the hypopharynx and larynx have low sensitivity and specificity. ${ }^{16,17}$ Twenty-four hour dual $\mathrm{pH}$ monitoring had been recommended as the gold standard for the diagnosis of LPR; however, nonacid or gas reflux mixed with liquid that could induce LPR symptoms would be missed in this diagnostic modality. $^{18}$

Recently, hypopharyngeal MII-pH (HMII-pH) monitoring with a specialized impedance catheter to measure LPR has been used to evaluate patients with symptoms of disease based on the established normative data. ${ }^{19}$ A certain number of NIPs diagnosed with GERD or misdiagnosed with SWD are supposed to suffer LPR. However, so far, the nature of LPR, particularly in NIPs, has not been investigated.

Therefore, this study aims to evaluate the nature of LPR and investigate the usefulness of pharyngeal BI values to assess SWD and GERD using HMII-pH in NIPs.

\section{Materials and Methods}

\section{Patients}

The study population in this retrospective study included 20 NIPs (mean age, $36.1 \pm 15.0$ years; age range, 13-64 years) who underwent MII-pH, HMII-pH, and laryngoscopy using the HSM at the Kurume University Hospital from December 2016 to April 2019. In preparation for study, medications for GERD (ie, proton pump inhibitors, prokinetics, and herbal medicine [Rikkunshito]) were stopped at least 3 days before study enrollment. The study protocol was approved by the Kurume University Ethical Committee (No. 16074). Informed consent was obtained from families of patients before starting the study.

\section{Measurement Techniques}

\section{Esophageal combined with pH-multichannel intralumi- nal impedance and hypopharyngeal multichannel intra- luminal impedance combined with $\mathrm{pH}$ monitoring}

Two multiple intraluminal impedance catheters with $2 \mathrm{pH}$ antimony electrodes and 7 impedance electrodes (patient's height $<150$ cm, CZPN-BG-57; $\geq 150$ cm, ZAN-BG-44; Diversatek, Highlands Ranch, CO, USA) were used for MII-pH. The catheter was inserted transnasally through the esophagus, and the $\mathrm{pH}$ sensor was fluoroscopically placed above the 2 vertebrae of the dia- 
phragm.

The catheter with $2 \mathrm{pH}$ probes in the hypopharynx and distal esophagus, and 2 pairs of impedance electrodes (ZAL-BL-56 and ZAI-BL-55; Diversatek ) each in the hypopharynx, proximal esophagus ( $2 \mathrm{~cm}$ and $4 \mathrm{~cm}$ distal to the upper esophageal sphincter), and distal esophagus $(3 \mathrm{~cm}$ and $5 \mathrm{~cm}$ proximal to the gastroesophageal junction) were used for HMII-pH. ${ }^{20}$ The blue visualpositioning band (hypopharyngeal $\mathrm{pH}$ sensor) was placed at the upper border of the cricopharyngeal muscle under endoscopic visualization. Additionally, an X-ray was taken to confirm the proper positioning of the probe. ${ }^{19}$ Data were uploaded from the recording device and analyzed using a dedicated software program (Bioview Analysis; Diversatek). All impedance tracings identified by software were individually reviewed by the same investigators on our standard protocol (D.M. and S.F.). ${ }^{4.8}$ Patients with a $\mathrm{pH}$ index (pHI) of $\geq 5 \%$ or $>70$ reflux episodes were diagnosed with pathological GERD. $^{21}$

The LPR event was defined as the retrograde transit of the bolus across all ring sets until it reached the hypopharynx. The diagnoses of acid and nonacid LPR were reached when the distal and hypopharyngeal $\mathrm{pH}$ were $\leq 4$ and $>4$, respectively. Full column reflux (FCR) was defined as reflux that reached the impedance site $2 \mathrm{~cm}$ distal to the upper esophageal sphincter, but not the hypopharyngeal ring set. Based on the established normative data of LPR and FCR, abnormal proximal exposure (APE; pathological LPR) was defined as an LPR of 1 or more events/24 hours and/ or an FCR of 5 or more events $/ 24$ hours. ${ }^{19}$

The mean 24-hour hypopharyngeal BI was calculated using the automatic calculating function (electronic ruler) in the software program.

\section{Laryngoscopy}

All NIPs underwent flexible endoscopic (Nagashima Medical Instruments Co, Ltd, Tokyo, Japan) evaluation of SWD using the HSM, as described previously. This method consists of 4 parameters: (1) salivary pooling at the vallecula and pyriform sinuses; (2) glottal closure reflex induction by touching the epiglottis or arytenoid with the endoscope; (3) swallowing reflex initiation assessed by "white-out," defined as the period during which the endoscopic image is obscured owing to pharyngeal closure, timing; and (4) pharyngeal clearance after swallowing blue-dyed water. Each parameter is scored from 0 to 3 on a 4-point scale ( 0 , normal; 1 , mildly impaired; 2 , moderately impaired; and 3, severely impaired).

The Hyodo score is expressed as the sum of scores for each of the 4 parameters and ranges from 0 to 12 . Patients with a score $<$
NIP: 20

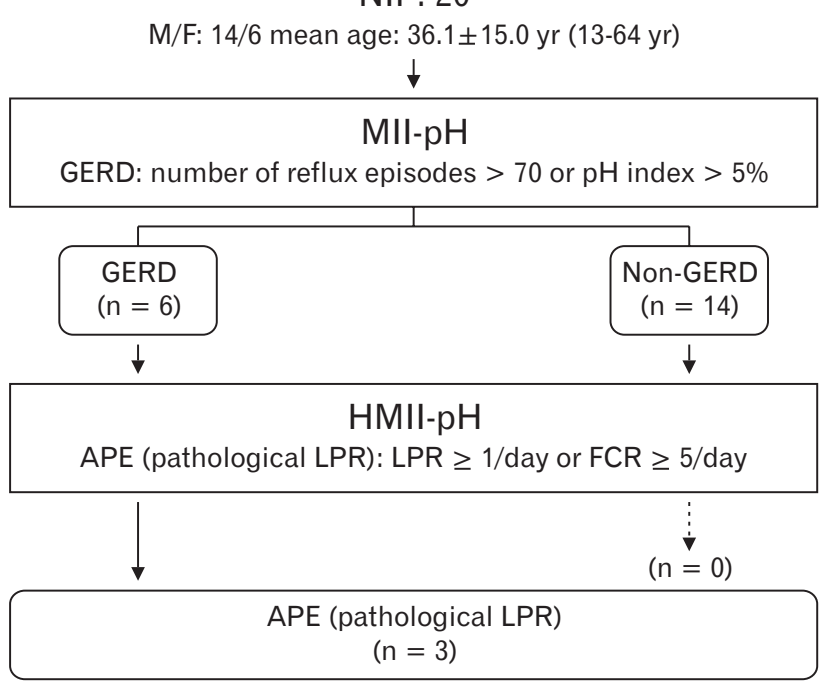

Figure 1. Of the 20 neurologically impaired patients (NIPs) who underwent multichannel intraluminal impedance and $\mathrm{pH}$ (MII-pH) and hypopharyngeal MII-pH (HMII-pH) monitoring, 6 were diagnosed with gastroesophageal reflux disease (GERD) by MII-pH monitoring; among them, 3 were diagnosed with abnormal proximal exposure (APE) based on the findings of the full column reflux (FCR) by HMII-pH monitoring. M, male; F, female; LPR, laryngopharyngeal reflux.

5 were defined as normal swallowing and would be allowed oral intake without limitations, whereas those with $\geq 8$ were judged as severe SWD and would not be allowed any oral intake. A score of $\geq 5$ was defined as SWD. ${ }^{10}$

\section{Statistical Methods}

All statistical analyses were performed using the JMP Pro 13 software program (SAS Institute Inc, Cary, NC, USA). The Mann-Whitney $U$ test was conducted for comparing the MII-pH and HMII-pH parameters (pHI, bolus exposure index, number of reflux episodes [total, acid, and nonacid], and number of FCR episodes [total, acid, and nonacid]) in all NIPs and the hypopharyngeal BI values between NIP with $\geq 5$ and $<5$ Hyodo scores. Spearman's correlation analysis was used to assess the correlation between the hypopharyngeal BI and Hyodo score. A receiver operator characteristic curve was used to determine the optimal cutoff BI value to identify SWD. Values of $P<0.05$ were statistically significant.

\section{Results}

Of the 20 NIPs who underwent MII-pH and HMII-pH 
monitoring, 6 were diagnosed with GERD by MII-pH monitoring; among them, 3 were determined as APE based on the finding of FCR by HMII-pH (Fig. 1). No significant differences in pHI, bolus exposure index and the numbers of reflux and FCR episodes (total, acid, and nonacid) were observed between MII-pH and HMII-pH (Table).

Among the 20 NIPs who underwent flexible endoscopic evaluation of HSM, 15 presented with a score of $\geq 5$ and 5 with $<5$. Furthermore, 5 (33\%) NIPs with a score of $\geq 5$ and 1 (20\%) with $<5$ were diagnosed with pathological GERD. All 3 patients with APE presented with SWD.

Table. Comparison of Each Parameter Between Multichannel Intraluminal Impedance and $\mathrm{pH}$ and Hypopharyngeal Multichannel Impedance and $\mathrm{pH}$ Measurements

\begin{tabular}{lccc}
\hline \multicolumn{1}{c}{ Parameters } & $\begin{array}{c}\text { MII-pH } \\
(\mathrm{n}=20)\end{array}$ & $\begin{array}{c}\text { HMII-pH } \\
(\mathrm{n}=20)\end{array}$ & P-value \\
\hline pH Index (esophagus) (\%) & $4.2 \pm 8.6$ & $3.4 \pm 7.0$ & 0.780 \\
Bolus exposure index (\%) & $0.8 \pm 1.3$ & $0.8 \pm 0.7$ & 0.950 \\
No. of reflux episodes (total) & $29.6 \pm 24.7$ & $31.8 \pm 20.8$ & 0.762 \\
No. of reflux episodes (acid) & $19.0 \pm 18.5$ & $17.7 \pm 17.1$ & 0.819 \\
No. of reflux episodes (non-acid) & $10.2 \pm 11.6$ & $13.6 \pm 11.6$ & 0.417 \\
No. of FCR episodes (total) & $6.0 \pm 9.7$ & $2.1 \pm 5.2$ & 0.123 \\
No. of FCR episodes (acid) & $4.1 \pm 7.4$ & $1.9 \pm 4.9$ & 0.274 \\
No. of FCR episodes (non-acid) & $1.3 \pm 2.7$ & $0.2 \pm 0.4$ & 0.071 \\
\hline
\end{tabular}

MII-pH, multichannel intraluminal impedance and $\mathrm{pH}$; HMII-pH, hypopharyngeal multichannel intraluminal impedance and $\mathrm{pH} ; \mathrm{FCR}$, full colum reflux.

Values are expressed as mean $\pm \mathrm{SD}$.

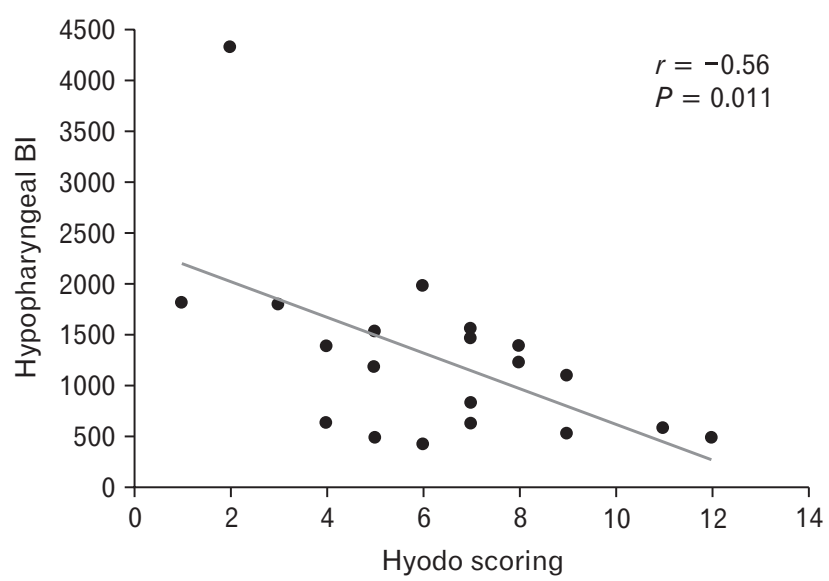

The hypopharyngeal BI values in NIP with a score of $\geq 5$ were significantly lower value than those with $<5$ in HSM $(1015 \pm$ $519 \Omega$ vs $1851 \pm 1294 \Omega ; P=0.047)$.

A significant moderate negative correlation $(r=-0.56, P=$ 0.011 ) was observed between the hypopharyngeal BI values and Hyodo scores. The optimal cutoff value of the hypopharyngeal BI to identify SWD was set at $1552 \Omega$. The corresponding sensitivity and specificity were 0.93 and 0.50 , respectively. The area under the curve was 0.74 (Fig. 2). A significant moderate negative correlation $(r=-0.49, P=0.032)$ was observed when the outlier was excluded (hypopharyngeal BI value, $4322 \Omega$ ); however, the corresponding cutoff hypopharyngeal BI value was not available.

The typical waveform in a non-GERD NIP with SWD is shown in Figure 3. Despite the absence of any LPR event, the hypopharyngeal BI value was very low. The patient had a history of recurrent aspiration pneumonia, was diagnosed with SWD based on HSM, and underwent laryngotracheal separation (Fig. 3).

\section{Discussion}

Although several previous studies have reported the prevalence of LPR in the neurologically normal population ${ }^{22,23}$ its exact prevalence is unknown due to the lack of a gold standard diagnostic test. On the other hand, the diagnosis of LPR by MII-pH/HMII-pH is becoming increasingly accepted according to a recent systematic review. ${ }^{24}$ The studies by Suzuki et $\mathrm{al}^{25}$ and Hoppo et al, ${ }^{26}$ which analyzed the LPR by HMII-pH, reported that $73-85 \%$ of patients with LPR symptoms had APE (pathological LPR), whereas LPR

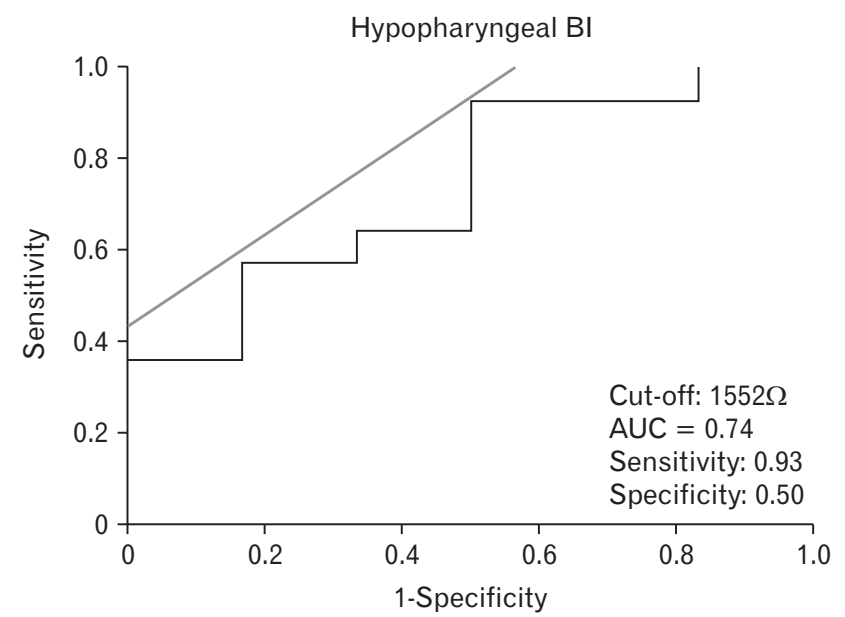

Figure 2. Hypopharyngeal impedance baseline values negatively correlated with Hyodo scores. The optimal cutoff value of the baseline impedance (BI) in Z1 (impedance channel positioned at hypopharynx) to identify the presence of swallowing dysfunction was determined to be $1552 \Omega+$. The corresponding sensitivity was 0.93 , specificity was 0.50 , and the area under the curve (AUC) was 0.74 . 


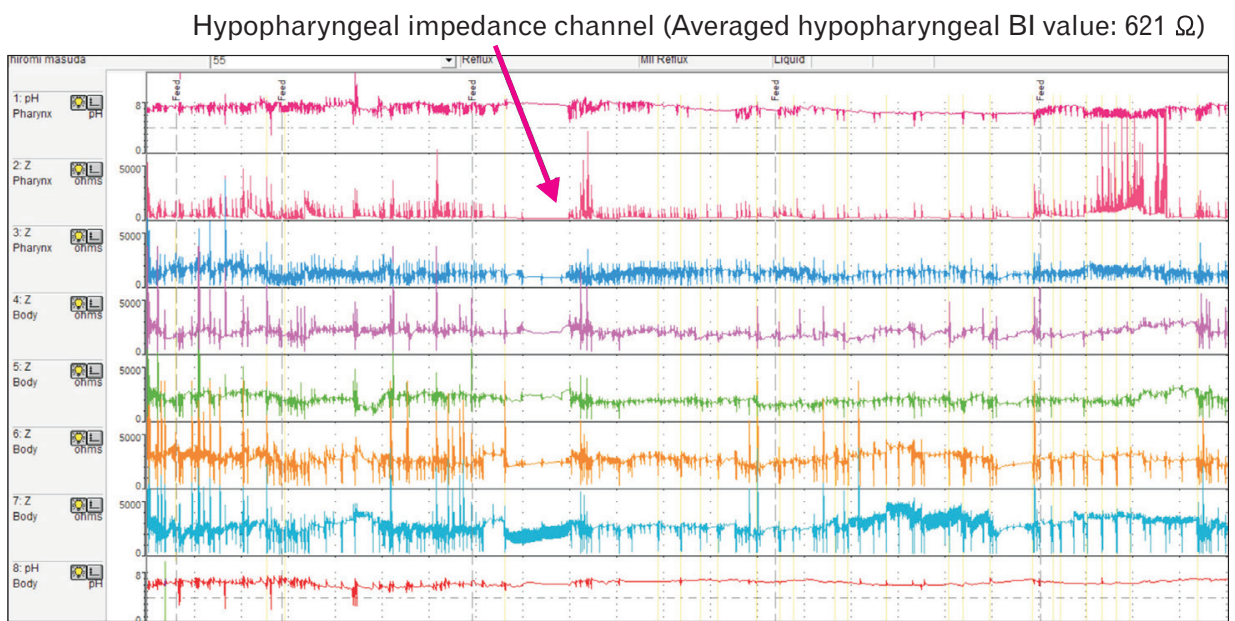

Figure 3. Hypopharyngeal multichannel intraluminal impedance and $\mathrm{pH}$ (HMII-pH) waveform showing no laryngopharyngeal reflux event and very low impedance baseline value in the hypopharyngeal channel. BI, baseline impedance. is reported to be extremely rare in healthy asymptomatic subjects. ${ }^{19}$

In the present study, few patients were diagnosed with pathological LPR, according to the criteria of FCR; all NIPs with pathological LPR presented with pathological nonacid LPR and accounted for half of the GERD cases. In another study, the LPR event or FCR in patients with LPR symptoms was found to occur almost exclusively in the upright position, and many of the patients had a complete symptomatic response with antireflux surgery despite a normal preoperative DeMeester score. ${ }^{19}$ As an explanation for the relationship between LPR and the upright position, it was proposed that this position impacts the competency of the lower esophageal sphincter. ${ }^{27}$ Based on this theory, LPR is less likely to occur in NIPs because they are always in the supine position. Furthermore, it was reported that the LPR event occurs in the supine position in association with GER. In the present study, the pathological LPR in all NIPs was accompanied by GERD, indicating that LPR in these patients may present with characteristics associated with GER. However, the present study comprised of 20 NIPs only, and was not able to fully clarify the relationships between NIPs and pathological acid LPR (LPR diagnosed by a drop in the hypopharyngeal $\mathrm{pH}$ ) and LPR and posture.

To the best of our knowledge, no study has compared the parameters between MII-pH and HMII-pH so far. The present study showed no significant difference in the key parameters between MII-pH and HMII-pH, suggesting that HMII-pH can be used to evaluate the presence of GERD, in addition to LPR and FCR, in NIPs.

However, the placement of $\mathrm{pH}$ and impedance sensors on the catheters differs considerably between MII-pH and HMII-pH. Moreover, due to the lack of catheters for HMII-pH in children, it cannot be used to accurately evaluate the reflux episode in the lower esophagus of children. Hence, the launch of an HMII-pH catheter specialized for children is desirable. Currently, MII-pH is more useful than HMII-pH in evaluating the reflux episodes in the lower esophagus or on the proton pump inhibitor in children.

Some studies adopted the BI to assess esophageal mucosal damage in the lower esophagus ${ }^{28,29}$; alternatively, there has been no attempt to diagnose SWD by adopting this method. To the best of our knowledge, this is the first study to evaluate pharyngeal salivary retention using BI. The averaged 24-hour BI value of the hypopharyngeal impedance channel was determined to evaluate the SWD. A significantly negative correlation between the hypopharyngeal BI value and Hyodo score was noted, indicating that hypopharyngeal BI evaluation may aid in the assessment of SWD in NIP. Furthermore, we attempted to determine the cutoff value of the hypopharyngeal BI. However, the calculated cutoff BI value, $1552 \Omega$, was higher than expected, and its specificity (50\%) was insufficient. A larger study with bigger sample size is necessary to obtain a more accurate cutoff value.

Nevertheless, the present study has several limitations, such as the small study population and the wide age range of the enrolled NIP, which may have affected the results. Further studies using a larger population are necessary to elucidate a more detailed pathophysiology of LPR and the usefulness of hypopharyngeal BI for diagnosing SWD in NIPs.

In conclusion, the present study demonstrated the presence of pathological LPR in NIPs and indicated that some of these patients may be diagnosed with GERD or misdiagnosed with SWD, despite the small number of neurologically normal subjects. In addition, our findings indicated that hypopharyngeal BI may be useful for evaluating SWD in NIP. Considering the difficulties in performing examinations in NIP, HMII-pH may prove to be 
a useful technique for the simultaneous evaluation of SWD, LPR, and GERD in these patients.

Acknowledgements: : The authors thank ENAGO for editing this manuscript.

\section{Financial support: None.}

\section{Conflicts of interest: None.}

Author contributions: Daisuke Masui and Suguru Fukahori designed the research study, analyzed the data, and wrote the paper; Minoru Yagi and Yoshiaki Tanaka designed the research study and wrote the paper; Shinji Ishii and Naoki Hashizume analyzed the data; and Nobuyuki Saikusa, Naruki Higashidate, Saki Sakamoto, Shiori Tsuruhisa, and Hirotomo Nakahara performed the research.

\section{References}

1. Stallings VA, Cronk CE, Zemel BS, Charney EB. Body composition in children with spastic quadriplegic cerebral palsy. J Pediatr 1995;126(5 pt 1):833-839.

2. Ceriati E, De Peppo F, Ciprandi G, Marchetti P, Silveri M, Rivosecchi M. Surgery in disabled children: general gastroenterological aspects. Acta paediatr Suppl 2006;95:34-37.

3. Plioplys AV, Kasnicka I, Lewis S, Moller D. Survival rates among children with severe neurologic disabilities. South Med J 1998;91:161-172.

4. Fukahori S, Yagi M, Ishii S, et al. Analyses of the relationship between a 'number of reflux episodes' exceeding 70 and the $\mathrm{pH}$ index in neurologically impaired children by evaluating esophageal combined $\mathrm{pH}$-multichannel intraluminal impedance measurements. Scand J Gastroenterol 2018;53:519-526.

5. Fukahori S, Yagi M, Ishii S, et al. A baseline impedance analysis in neurologically impaired children: a potent parameter for estimating the condition of the esophageal mucosa. Neurogastroenterol Motil 2017;29.

6. Fukahori S, Yagi M, Ishii S, et al. Laparoscopic Nissen fundoplication mainly reduces the volume of acid reflux and potentially improves mucosal integrity up to the middle esophagus in neurologically impaired children detected by esophageal combined $\mathrm{pH}$-multichannel intraluminal impedance measurements. J Pediatr Surg 2016;51:1283-1287.

7. Fukahori S, Asagiri K, Ishii S, et al. Pre and post-operative evaluation of gastroesophageal reflux and esophageal motility in neurologically impaired children using combined $\mathrm{pH}$-multichannel intraluminal impedance measurements. Pediatr Surg Int 2013;29:545-551.

8. Masui D, Fukahori S, Ishii S, et al. The assessment of the esophageal motility of children with esophageal disorders by the detailed observation of the $\mathrm{pH}$-multichannel intraluminal impedance waveform and baseline impedance: screening test potential. Esophagus 2019;16:133-140.

9. Morgan AT, Dodrill P, Ward EC. Interventions for oropharyngeal dysphagia in children with neurological impairment. Cochrane Database Syst Rev 2012;10:CD009456.
10. Chiba Y, Sano D, Ikui Y, Nishimura G, et al. Predictive value of the Hyodo score in endoscopic evaluation of aspiration during swallowing. Auris Nasus Larynx 2018;45:1214-1220.

11. Sakamoto T, Horiuchi A, Makino T, Kajiyama M, Tanaka N, Hyodo M. Determination of the cut-off score of an endoscopic scoring method to predict whether elderly patients with dysphagia can eat pureed diets. World J Gastrointest Endosc 2016;8:288-294.

12. Koufman JA, Amin MR, Panetti M. Prevalence of reflux in 113 consecutive patients with laryngeal and voice disorders. Otolaryngol Head Neck Surg 2000;123:385-388.

13. Koufman JA. Laryngopharyngeal reflux is different from classic gastroesophageal reflux disease. Ear Nose Throat J 2002;81(9 suppl 2):7-9.

14. Harding SM, Guzzo MR, Richter JE. The prevalence of gastroesophageal reflux in asthma patients without reflux symptoms. Am J Respir Crit Care Med 2000;162:34-39.

15. Hoppo T, Komatsu Y, Jobe BA. Gastroesophageal reflux disease and patterns of reflux in patients with idiopathic pulmonary fibrosis using hypopharyngeal multichannel intraluminal impedance. Dis Esophagus 2014;27:530-537.

16. Vaezi MF, Hicks DM, Abelson TI, Richter JE. Laryngeal signs and symptoms and gastroesophageal reflux disease (GERD): a critical assessment of cause and effect association. Clin Gastroenterol Hepatol 2003;1:333-344.

17. Hickson C, Simpson CB, Falcon R. Laryngeal pseudosulcus as a predictor of laryngopharyngeal reflux. Laryngoscope 2001;111:1742-1745.

18. Desjardin M, Roman S, des Varannes SB, et al. Pharyngeal $\mathrm{pH}$ alone is not reliable for the detection of pharyngeal reflux events: a study with oesophageal and pharyngeal $\mathrm{pH}$-impedance monitoring. United European Gastroenterol J 2013;1:438-444.

19. Hoppo T, Sanz AF, Nason KS, et al. How much pharyngeal exposure is "normal"? Normative data for laryngopharyngeal reflux events using hypopharyngeal multichannel intraluminal impedance (HMII). J Gastrointest Surg 2012;16:16-24; discussion 24-25.

20. Komatsu Y, Kelly LA, Zaidi AH, et al. Hypopharyngeal pepsin and sep70 as diagnostic markers of laryngopharyngeal reflux: preliminary study. SurgEndosc 2015;29:1080-1087.

21. Fukahori S, Kawahara H, Oyama T, et al. Standard protocol devised by the Japanese pediatric impedance working group for combined multichannel intraluminal impedance-pH measurements in children. Surg Today 2020;50:664-671.

22. Koufman JA, Aviv JE, Casiano RR, Shaw GY. Laryngopharyngeal reflux: position statement of the committee on speech, voice, and swallowing disorders of the American academy of otolaryngology-head and neck surgery. Otolaryngol Head Neck Surg 2002;127:32-35.

23. Reulbach TR, Belafsky PC, Blalock PD, Koufman JA, Postma GN. Occult laryngeal pathology in a community-based cohort. Otolaryngol Head Neck Surg 2001;124:448-450.

24. Lechien JR, Akst LM, Hamdan AL, et al. Evaluation and management of laryngopharyngeal reflux disease: state of the art review. Otolaryngol Head Neck Surg 2019;160:762-782.

25. Suzuki T, Seki Y, Okamoto Y, Hoppo T. Hypopharyngeal multichannel intraluminal impedance leads to the promising outcome of antireflux 
surgery in Japanese population with laryngopharyngeal reflux symptoms. Surg Endosc 2018;32:2409-2419.

26. Hoppo T, Zaidi AH, Matsui D, et al. Sep70/pepsin expression in hypopharynx combined with hypopharyngeal multichannel intraluminal impedance increases diagnostic sensitivity of laryngopharyngeal reflux. Surg Endosc 2018;32:2434-2441.

27. Hoppo T, Komatsu Y, Nieponice A, Schrenker J, Jobe BA. Toward an improved understanding of isolated upright reflux: positional effects on the lower esophageal sphincter in patients with symptoms of gastroesoph- ageal reflux. World J Surg 2012;36:1623-1631.

28. Borrelli O, Salvatore S, Mancini V, et al. Relationship between baseline impedance levels and esophageal mucosal integrity in children with erosive and non-erosive reflux disease. Neurogastroenterol Motil 2012;24:828-e394.

29. Frazzoni M, Savarino E, de Bortoli N, et al. Analyses of the post-reflux swallow-induced peristaltic wave index and nocturnal baseline impedance parameters increase the diagnostic yield of impedance-pH monitoring of patients with reflux disease. Clin Gastroenterol Hepatol 2016;14:40-46. 